\title{
Philosophiques
}

\section{Du psychologisme au platonisme : l'évolution de Reinach sur les fondements du droit}

\section{Hamid Taieb}

Volume 46, numéro 1, printemps 2019

URI : https://id.erudit.org/iderudit/1062019ar

DOI : https://doi.org/10.7202/1062019ar

Aller au sommaire du numéro

\section{Éditeur(s)}

Société de philosophie du Québec

ISSN

0316-2923 (imprimé)

1492-1391 (numérique)

Découvrir la revue

Citer cet article

Taieb, H. (2019). Du psychologisme au platonisme : l'évolution de Reinach sur les fondements du droit. Philosophiques, 46(1), 207-227.

https://doi.org/10.7202/1062019ar
Résumé de l'article

Reinach est connu pour défendre une théorie a priori du droit civil. Cette position, qui se dit inspirée de Husserl, est usuellement qualifiée de " platonisme » dans la littérature secondaire. Elle se comprend comme intuition des essences, projet de phénoménologie eidétique que Reinach fait remonter à Platon. Une position qui est rejetée par Reinach en philosophie du droit est le " psychologisme ", thèse tendant à expliquer le droit par référence à la psyché. L'hostilité de Reinach à l'égard du psychologisme semble être une hostilité à l'égard de lui-même : en tant qu'élève de Lipps, Reinach avait écrit une thèse sur la notion de causalité en droit pénal, dans laquelle il faisait jouer à la psychologie un rôle fondateur pour le droit. Dans mon exposé, je retracerai l'évolution de Reinach sur ces questions, en repartant de sa thèse et des travaux de Lipps sur lesquels elle se base pour la comparer aux écrits reinachiens plus tardifs et leur rapport tant à Platon qu’à Husserl. 


\title{
Du psychologisme au platonisme: l'évolution de Reinach sur les fondements du droit
}

\author{
HAMID TAIEB \\ Université de Hambourg
}

\begin{abstract}
RÉSUMÉ. - Reinach est connu pour défendre une théorie a priori du droit civil. Cette position, qui se dit inspirée de Husserl, est usuellement qualifiée de «platonisme » dans la littérature secondaire. Elle se comprend comme intuition des essences, projet de phénoménologie eidétique que Reinach fait remonter à Platon. Une position qui est rejetée par Reinach en philosophie du droit est le «psychologisme», thèse tendant à expliquer le droit par référence à la psyché. L'hostilité de Reinach à l'égard du psychologisme semble être une hostilité à l'égard de lui-même: en tant qu'élève de Lipps, Reinach avait écrit une thèse sur la notion de causalité en droit pénal, dans laquelle il faisait jouer à la psychologie un rôle fondateur pour le droit. Dans mon exposé, je retracerai l'évolution de Reinach sur ces questions, en repartant de sa thèse et des travaux de Lipps sur lesquels elle se base pour la comparer aux écrits reinachiens plus tardifs et leur rapport tant à Platon qu'à Husserl.
\end{abstract}

\begin{abstract}
Reinach is known for his defence of an a priori theory of civil law. His position, which is meant to be inspired by Husserl, is usually said to be "Platonist" in the secondary literature. It is understood as an intuition of essences, and this project of eidetic phenomenology would originate in Plato according to Reinach. A position which is rejected by Reinach in philosophy of law is "psychologism", which tends to explain laws by reference to psychical phenomena. The hostility of Reinach against psychologism seems to be a hostility against himself: as a student of Lipps, Reinach wrote a dissertation on the notion of causality in criminal law and gave in this text a founding role to psychology with respect to law. In my paper, I follow the evolution of Reinach on these questions, by starting from his dissertation and the works of Lipps on which it is based in order to compare it to the later Reinachian writings and their relation both to Plato and Husserl.
\end{abstract}

\section{Introduction}

Peut être qualifiée de "psychologiste", en première approximation, toute théorie visant à analyser certains phénomènes non physiques tels que la logique, la sémantique, le droit, etc., en termes d'activités psychiques, que celles-ci soient considérées en général, ou comme appartenant à une espèce donnée, par exemple l'être humain, voire à un individu particulier ${ }^{1}$. Le psychologisme est usuellement associé à la question des fondements de la logique. Pour un défenseur du psychologisme, les lois logiques ne sont rien d'autre que l'expression de régularités dans les enchaînements de la pensée

1. Sur la diversité des usages du terme "psychologisme», et ce dès son apparition dans les discussions philosophiques, cf. Kusch 1995, et Dewalque \& Gyemant 2015. 
humaine. $\mathrm{Si}$ « $p->q$, et $p$, donc $q$ », c'est parce que les êtres humains qui pensent $p \rightarrow q$, et $p$, en concluent que $q$. Comme le souligne Husserl, le psychologisme compris en ce sens est en fait un "anthropologisme» et a des conséquences relativistes quant à la logique (Husserl I975, I30.I8-I3I.32). Cette position a été contestée par divers auteurs, à commencer par Husserl lui-même, dont l'anti-psychologisme est usuellement considéré comme une prise de distance à l'égard de son maître Brentano. D'après Husserl, la logique n'a aucune dépendance à l'égard de la psychologie humaine. C'est au contraire parce que les humains sont doués de raison qu'ils parviennent (le plus souvent) à appliquer correctement les enchaînements requis par les lois logiques. Si les humains avaient été irrationnels, cela n'aurait en rien changé la validité logique du modus ponens ${ }^{2}$. Le reproche de "psychologisme » en logique a néanmoins eu divers sens. Ainsi, on trouve chez Reinach, élève de Husserl, une variante non anthropologique de psychologisme: sont psychologistes ceux qui souhaitent «expliquer les règles de la logique par retour sur des vécus plutôt que de les élucider par leur propre analyse et de les rendre évidents", par exemple ceux qui affirment qu' "un vécu de "nécessité de pensée" peut nous offrir une garantie de la validité des lois objectives de la logique» (Reinach I989 [I9I3a], I83.I3-20). Ici, il n'est pas fait mention de psychologie humaine, de sorte que la critique ne porte pas sur de quelconques conséquences relativistes. Au-delà de cette différence, pour Husserl, de même que pour Reinach, les lois de la logique ne sont pas des lois réelles, mais «idéales» (Husserl I975, 76.4-80.I7). Les entités idéales n'incluent pas seulement les lois logiques, mais aussi les nombres, ou les genres et les espèces, appelées aussi "essences". Par contraste aux entités réelles, les «idéalités » sont atemporelles et Husserl, dans les Recherches logiques, semble leur accorder une certaine existence ${ }^{3}$. En tous les cas, sa position sur les objets idéaux, et donc les lois de la logique, a été interprétée comme une variante du "platonisme ${ }^{4}$.

2. Je suis la présentation faite en Chisholm I960, p. I3-I4. Il serait impossible de présenter ici de manière exhaustive la genèse de la querelle du psychologisme, tant les acteurs de cet épisode majeur de l'histoire de la philosophie sont nombreux. Il convient toutefois de noter que l'ancrage de Husserl dans l'école de Brentano, ainsi que sa prise de distance à l'égard de son maître sur cette question constituent des éléments-clefs de la narration habituelle, bien que l'attribution du psychologisme à Brentano ne soit pas si évidente, comme en attestent Dewalque \& Gyemant 20I 5 .

3. Cf. notamment Husserl I984, I30.9-I0.

4. La thèse d'un platonisme de Husserl, bien que souvent défendue, a parfois aussi été contestée, notamment par Seron 20I2, qui rapproche la théorie husserlienne de celle d'Aristote, du moins pour ce qui est des significations comprises comme objets idéaux. Si Husserl devait s'avérer ne pas être platonicien, la position de Reinach, étant admis qu'elle soit bel et bien platonicienne, ne serait dès lors pas héritée de son maître, mais constituerait un développement philosophique propre. Ce que cet article souhaite avant tout soutenir, c'est que Reinach s'inspire pour son propre platonisme de celui que Husserl prétend endosser. Afin de régler la question de savoir si Husserl et/ou Reinach sont effectivement platoniciens, il conviendrait de 
Un aspect bien moins célèbre des querelles autour du psychologisme est l'apparition de cette dernière notion dans les discussions sur les fondements du droit. De même que la logique ne doit rien à la psychologie pour sa validité, la théorie du droit ne devrait pas — du moins pas systématiquement - se rapporter aux activités psychiques humaines pour l'élucidation de la nature des entités juridiques. Cette critique du psychologisme en philosophie du droit a été défendue par Reinach, dans son texte Les fondements a priori $d u$ droit civil de I9I3. Reinach était un membre influent du groupe des phénoménologues dits "de Munich et Göttingen ", qui constituait le premier réseau de défense et de développement des thèses de Hus$\operatorname{serl}^{5}$. Or une particularité de ce groupe, outre son attachement au réalisme métaphysique, est son attrait pour la théorie husserlienne des objets idéaux, notamment des essences et de leurs relations. Platon joue ainsi un rôle important aux yeux de ces auteurs, comme en attestent notamment les «Bemerkungen über das Wesen, die Wesenheit und die Idee » de Jean Héring (de I92I), l'article "Ideelle Existenz» de Maximilian Beck (de I929) ou encore «Über das Wesen der Idee» écrit par Herbert Spiegelberg (en I930). Reinach s'est lui aussi longuement intéressé à Platon, consacrant notamment à ce dernier un cours au semestre d'été I9 Io (Reinach 2000). Reinach en vient ainsi à appliquer la Wesenschau en philosophie du droit: comme pour tout autre domaine, c'est dans les essences elles-mêmes et leurs relations que l'on trouve les fondements du droit. Il y a donc une théorie «eidétique » des entités juridiques (d'cĩosos, «essence » ou «Idée ») et la position de Reinach en philosophie du droit est elle aussi souvent qualifiée de "platonicienne» (Reinach I989 [1913a], I83.13-23)6.

Le psychologisme logique était notamment défendu par Theodor Lipps $^{7}$, philosophe et psychologue influent du tournant du $\mathrm{xx}^{\mathrm{e}}$ siècle, et dont beaucoup de phénoménologues de Munich et Göttingen avaient suivi les enseignements. Or la personne que Reinach attaque lorsqu'il s'oppose au psychologisme juridique, c'est encore Lipps (Reinach I989 [I9I3a], I79.I9I 83.23). L'hostilité de Reinach à l'égard du psychologisme juridique semble être une hostilité à l'égard de lui-même: lorsqu'il était élève de Lipps, Rei-

se demander en quel sens Platon lui-même l'est, et ce afin d'avoir un terme fiable pour la comparaison. Il est clair que cette question ne saurait être tranchée ici.

5. Sur cette tradition, voir Salice 2015.

6. Pour l'attribution du platonisme, voir par exemple Smith I987. Pour son rejet, voir Baltzer-Jaray 2009 et, dans une moindre mesure, Dubois I995, notamment p. I03-I04. Il va de soi que ce qui vaut pour Reinach vaut aussi pour Héring, Beck et Spiegelberg: si Husserl devait s'avérer ne pas être platonicien, comme le défend notamment Seron 20I2, le platonisme de ces auteurs, étant admis qu'il en soit un, constituerait là aussi un développement philosophique propre.

7. Voir notamment Lipps I880, cité dans Chisholm I960, p. I3. Sur les rapports entre Lipps et Husserl concernant le psychologisme, voir Lavigne 20I 8. Sur Lipps plus généralement, voir les études récentes dans Depraz, Espagne \& Galland-Szymkowiak 2017 et 20 I 8. 
nach avait écrit une thèse sur la notion de causalité en droit pénal, dans laquelle il faisait jouer à la psychologie un rôle fondateur pour le droit. Si la position du jeune Reinach, ainsi que la critique qui s'en est suivie, est souvent évoquée pour des raisons dramaturgiques (reniement de l'enseignement du maître, etc.), il est plutôt rare de trouver des analyses précises sur ces thèmes ${ }^{8}$. Ainsi, la question de savoir ce qu'est exactement le psychologisme juridique de Lipps et, surtout, dans quelle mesure Reinach l'a adopté dans sa thèse n'est pas souvent traitée. Il est du coup difficile de savoir si les attaques plus tardives de Reinach contre Lipps sont des autocritiques.

Le présent article est destiné à élucider ces questions. Il commencera par étudier le rôle joué par la psychologie dans la thèse de Reinach et le rapport de ce texte à l'œuvre de Lipps. L'article présentera ensuite la critique faite par Reinach, dans ses écrits ultérieurs, du psychologisme juridique de Lipps. Il se penchera sur le platonisme de Reinach, en se demandant en quel sens Reinach accepterait de se voir attribuer le qualificatif de "platonicien». Ce faisant, l'article contribuera plus généralement à une meilleure compréhension des relations complexes entre philosophie et psychologie, au tournant du $\mathrm{XX}^{\mathrm{e}}$ siècle et peut-être au-delà.

\section{La psychologie dans la thèse de Reinach}

En I905, le jeune Reinach effectue, sous la tutelle de Lipps, une étude sur la notion de causalité en droit pénal positif allemand. Je ne m'intéresserai pas, dans ce qui suit, aux discussions centrales du traité, notamment à la critique faite par Reinach des principales interprétations de la causalité pénale, y compris la théorie de la causalité adéquate, ainsi qu'à la solution qu'il propose lui-même comme autre option. Mon intérêt portera exclusivement sur le rôle joué dans ce texte par la psychologie.

Reinach ouvre et clôt son traité par des considérations sur les rapports entre droit, philosophie et, surtout, psychologie. Plus précisément, il distingue "psychologie théorique du droit ", "psychologie pratique du droit", «herméneutique du droit» et finalement «philosophie du droit» (Reinach I989 [I905], I.3-7.30 et 43.I6-29). Celle-ci a une dimension éthique, puisque Reinach affirme que le but en est la découverte du droit juste. L'étude de Reinach portant sur le droit positif, la "philosophie du droit" au sens où il l'entend n'est quasiment pas invoquée dans les analyses, l'objectif n'étant pas de déterminer ce qui est juste ou injuste. La psychologie théorique du droit, quant à elle, est décrite comme "science des conditions d'émergence du droit en général et des systèmes juridiques individuels en particulier».

8. Reinach n'apparaît que brièvement dans une liste dans Kusch I995, p. 279. Pour une présentation de la philosophie du droit de Reinach, voir Cantegreil 2005 et Loidolt 20Io, qui se basent surtout sur Les fondements a priori $d u$ droit civil. Sur les grands courants de la pensée juridique allemande lors de la parution de ce texte, voir Halpérin 2008. 
Cette discipline explique la genèse du droit grâce à la psychologie, plus précisément la psychologie humaine. En effet, comme le soutient Reinach:

On peut se référer au fait que l'existence du droit présuppose certaines constitutions psychiques et rapports psychiques humains sous le règne desquels il a émergé, et qu'il revient indubitablement à la psychologie d'expliquer l'émergence du droit en général à partir de ces conditions psychologiques. On peut également remarquer qu'à côté des présuppositions du droit en général, il y a des présuppositions psychiques déterminées pour chaque système juridique particulier en tant que tel. Des intuitions morales, des préjugés, des opinions religieuses seraient par exemple évocables à ce titre; il revient à nouveau à la psychologie d'expliquer l'émergence des systèmes juridiques particuliers à partir de leurs conditions psychologiques. (Reinach I989 [I905], I.6-I6).

L'explication de Reinach semble être la suivante: le droit en général sert à réguler les interactions entre les humains, et il est établi sur la base de leurs «représentations ", au sens large, qui comprennent leurs croyances théoriques ainsi que leurs préférences éthiques, les droits particuliers sur la base de certains aspects plus précis des représentations de peuples déterminés. Or la connaissance des représentations humaines revient à la psychologie, de sorte que c'est elle qui est à même d'étudier les conditions d'émergence du droit. Il convient de noter que, pour Reinach, la psychologie semble capable d'expliquer la structure même des systèmes juridiques, qui ont apparemment une teneur commune basée, disons, sur des "invariants anthropologiques ", et contiennent certains éléments plus spécifiques dépendant de croyances ou d'intuitions morales propres à certaines cultures. Par exemple, de nombreuses sociétés humaines considèrent que les biens d'une personne doivent rester entre les mains de sa famille à sa mort; le droit consacre ce fait en prévoyant une succession automatique en ligne descendante et/ou ascendante, comprenant parfois une part minimale ne pouvant être léguée à des tiers. Il est possible de parler ici d'un psychologisme juridique anthropologique basé sur des considérations de psychologie génétique, au sens où la teneur des formations juridiques, bien que n'étant pas analysée à l'aide de notions psychologiques, est néanmoins expliquée à partir des activités psychiques humaines qui motivent l'émergence de ces formations.

Pour ce qui est de la psychologie pratique du droit, Reinach la définit comme la discipline qui dévoile les «faits et régularités psychiques » humains requis pour l'application du droit. Elle est dès lors avant tout destinée au juge. Reinach avance une série de cas en droit pénal dans lesquels le juge doit faire appel à ses connaissances en psychologie: premièrement, pour ce qui est de la fixation de la peine, le juge doit en estimer la dureté en tenant compte de toutes les circonstances, notamment celles qui sont "atténuantes ", ce qui requiert une connaissance des "instincts, motivations et passions" des êtres humains. Il doit par ailleurs déterminer quel type de peine (prison, amende) serait le plus efficace en fonction des différents justiciables. En ce qui concerne l'établissement des faits, le juge doit à nouveau 
faire appel à ses connaissances psychologiques, notamment lorsqu'il lui revient d'évaluer la véracité des témoignages, ceux-ci pouvant diverger de la réalité sans que les témoins eux-mêmes n'en aient conscience, suivant donc un processus psychique involontaire'. Ici, la psychologie humaine, bien que jouant un rôle instrumental important, ne détermine pas la teneur même des formations juridiques (ni analytiquement ni génétiquement), de sorte qu'il ne saurait être question de psychologisme.

Or ce n'est pas à la psychologie théorique ni à la psychologie pratique du droit que Reinach s'intéresse dans sa thèse, mais à l'importance des connaissances de la psychologie humaine pour l'herméneutique du droit. Selon Reinach, un système juridique n'est pas tant un ensemble de textes légaux, c'est-à-dire de signes linguistiques, que ce qui est exprimé ou signifié par ces signes. Ainsi, le juriste ou le juge, lorsqu'ils sont confrontés à un texte de loi, ne doivent pas se contenter, comme le lexicographe, de relever l'ambiguité des expressions, mais tenter de remonter d'elles à la reproduction de l'opinion ou «visée " (Meinung) du législateur (Reinach I989 [I905], 2.35-3.2I). Or, à un premier titre, la psychologie intervient dans l'explicitation du droit en ce que la visée du législateur porte souvent sur des objets qui sont psychiques: intention, négligence, etc. Ainsi, lorsqu'il reproduit l'opinion du législateur, le juriste prend en considération ces objets mêmes et se fait dès lors psychologue (Reinach I989 [1905], 4.25-5.5). En outre, la psychologie peut servir à désambiguïser certains énoncés juridiques, en expliquant les erreurs qui ont mené le législateur à utiliser tel signe plutôt que tel autre, plus habituel, pour exprimer une certaine idée, de même qu'elle peut servir à donner les raisons ou justifications de l'adoption d'une opinion à première vue étrange, rendant celle-ci plus vraisemblable (Reinach I989 [1905], 5.22-6.19). Or Reinach contraste fortement la prise en considération des objets psychologiques par le juriste et le cas où la psychologie sert à expliquer l'usage ambigu d'un signe ou le choix d'une opinion surprenante. Dans le premier cas, dit Reinach, la psychologie appartient à l'«essence » (Wesen) de la science juridique. L'idée est que, lorsqu'il interprète le droit, le juriste prend souvent pour objet des entités psychiques - volonté, etc. - , et se voit dès lors contraint, pour exercer son activité de juriste, d'être également psychologue (Reinach I989 [1905], 5·3-5). Comme le soutient Reinach, citant Pfänder, mais reprenant en fait une distinction de Brentano, il s'agit là de "psychologie descriptive ", comprise comme étude de la structure générale des actes mentaux et de leurs différentes classes, menée du point de vue de la première personne. Par contre, l'usage des connaissances psychologiques pour expliquer le choix de certaines expressions ou opinions revient à faire de la "psychologie explicative-causale ", c'est-à-dire génétique, qui inclut non seulement la causalité psycho-physiologique, mais 
aussi les relations de motivation ${ }^{10}$. Pour ce qui est de la prise en considération nécessaire d'entités psychiques par le juriste, il ne saurait à mon sens être question de psychologisme, puisqu'il ne s'agit pas tant d'élucider (que ce soit analytiquement ou génétiquement) la teneur de formations proprement juridiques - comme le contrat, l'obligation ou la prescription - à l'aide de la psychologie, que de constater l'intégration, en droit, de notions de psychologie. Par ailleurs, l'usage de la psychologie dans l'étude de l'opinion du législateur a un caractère instrumental, de sorte qu'il n'y a pas ici non plus de psychologisme.

En tout cas, la psychologie, plus précisément la psychologie humaine, intervient à plus d'un titre dans la thèse de Reinach. Elle lui sert en effet tant à expliquer l'émergence et la teneur des systèmes juridiques que l'interprétation et l'application du droit. Elle se réserve la part théorique dans l'analyse du droit, puisque la "philosophie du droit» est consacrée à l'éthique.

Qu'en est-il de Lipps dans la thèse de Reinach? Étrangement, Lipps n'est pas mentionné lorsqu'il s'agit d'établir les rapports généraux entre droit, philosophie et psychologie. Ainsi, Reinach ne l'invoque notamment pas lorsqu'il restreint la philosophie du droit à l'éthique, ni lorsqu'il oppose psychologie théorique et psychologie pratique du droit. Lipps est uniquement cité quand il est question de la mise en œuvre de la psychologie descriptive ou explicative-causale par le juriste. Ainsi, Reinach renvoie à Lipps afin d'élucider la nature de l' "intention " (Vorsatz) ou de discuter la sensation de «mienneté » d'une action dépendante de la volonté du sujet (Reinach I989 [1905], 30.19-35 et 4I.35-42.I). Par ailleurs, Reinach affirme que l'interprétation identique, par le juriste, des mêmes expressions utilisées par le législateur dans des circonstances similaires est justifiée par la règle lippséenne de la «fidélité à soi » (Reinach I989 [I905], 6.3 8-7.4).

Or, indépendamment des références explicites de Reinach, il semble que Lipps fasse jouer un rôle important à la psychologie dans sa théorie du droit. Ainsi, il soutient que la "peine » est destinée à faire prendre conscience de son mauvais comportement au criminel et de permettre ainsi une réconciliation avec la victime. La "peine» n'est pas tant une «action externe» qu'une « réaction interne » à l'injustice, plus précisément l'« exigence » émanant de la victime qu'il y ait une prise de conscience morale chez le criminel. Cette exigence, dit Lipps, est incluse dans le concept même de peine et est donc présupposée dans tous nos jugements et pratiques punitifs (Lipps I 899 , p. 290 et 294$)^{11}$.

Par ailleurs, Lipps affirme que le «droit» (Recht) auquel un criminel porte atteinte n'est ni le «concept de droit» (Begriff des Rechtes) ni une quelconque «disposition légale en vigueur» (geltende Rechtsbestimmungen),

10. Voir Reinach I989 [I905], 7.23-24, citant Pfänder I904. Pour l'origine de l'opposition, voir Brentano I982.

11. Pour de plus longs développements sur la notion de peine, voir Lipps I906b. 
mais la conscience de ses droits chez l'humain (menschliche Rechtsbewusstsein). Selon Lipps, s'il ne renvoie pas à cette conscience, le terme "droit » (Recht) est vide de sens (Lipps I 899, p. 293).

On trouve également chez lui la thèse voulant que certains droits soient explicables en termes de volonté. Plus précisément, il affirme que les droits naturels sont en vérité des «souhaits naturels » humains, ce qui explique leur objectivité (Lipps I906a, p. 210).

Lipps soutient en outre que la promesse est l'extériorisation de la volonté d'effectuer une action en faveur d'autrui. L' «obligation» (Verpflichtung), quant à elle, est l'acte de volonté reconnu par autrui et retourné chez la personne qui l'a émis «rehaussé de l'intérêt de l'autre» (Lipps I906a, p. 210).

Ainsi, des notions juridiques tout à fait déterminantes sont analysées par Lipps au moyen de concepts issus de la psychologie descriptive, soit ceux de "réaction interne ", d' "exigence ", de "conscience ", de "souhait» ou de "volonté». Qui plus est, la psychologie dont il parle semble être la psychologie humaine ${ }^{12}$. Ainsi, Lipps fonde définitionnellement le droit sur la psychologie, au sens où il analyse le contenu des notions juridiques en termes psychologiques humains. On pourrait dès lors parler, en ce qui concerne Lipps, d'un psychologisme juridique anthropologique fondé sur la psychologie descriptive ${ }^{13}$. Reinach, dans sa thèse, ne semble pas procéder de la sorte.

Avant d'en venir aux Fondements a priori $d u$ droit civil et à la critique du psychologisme juridique, résumons dès lors les positions de Reinach et de Lipps. Reinach procède à une réflexion de large portée sur les rapports entre le droit et la psychologie, plus précisément la psychologie humaine, distinguant notamment psychologie théorique et pratique du droit, faisant intervenir la psychologie dans l'herméneutique juridique et insistant sur la présence d'un grand nombre de notions psychologiques en droit. Pour Reinach, la psychologie épuise l'explication théorique du droit, tant pour ce qui est de son émergence que de la teneur des systèmes juridiques, la philosophie du droit étant réservée à l'éthique. Reinach fait certes fortement dépendre le droit de la psychologie, sans doute sous l'influence diffuse de Lipps, mais il ne le fait pas au sens où il analyserait le contenu des notions juridiques en termes psychologiques. Lipps, par contraste, ne semble pas avoir de réflexion géné-

12. Voir notamment l'insistance sur l'être humain dans Lipps I906a, et surtout Lipps I 899 .

13. Pour l'usage de l'opposition brentanienne évoquée ci-dessus entre psychologie descriptive et psychologie génétique dans l'étude du psychologisme, voir Dewalque \& Gyemant 20I 5 . À noter que Reinach lui-même utilise ponctuellement l'opposition, mais relativement au psychologisme logique: dire que l'étude des actes de jugement relève de la logique revient à assimiler la logique à la psychologie descriptive, soutenir que l'inférence est un processus psychique produit une confusion entre logique et psychologie génétique; toutefois, l'analyse en termes de psychologie génétique porte sur des connexions entre éléments fixés préalablement par la psychologie descriptive, en l'occurrence des connexions entre des jugements (Reinach I989 [I9I3b], 459.33-460.3). 
rale sur les rapports entre droit et psychologie. Cela ne l'empêche néanmoins pas d'analyser le contenu de certains concepts juridiques à l'aide de notions psychologiques telles que "conscience ", "volonté », etc. On trouve ainsi chez Lipps, contrairement à ce qui est le cas chez Reinach, un psychologisme juridique anthropologique basé sur la psychologie descriptive. Reinach, dans sa thèse, peut toutefois lui aussi être qualifié de "psychologiste»: on décèle dans ce texte de jeunesse un psychologisme anthropologique basé sur des considérations de psychologie génétique, au sens où la teneur des formations juridiques, pour Reinach, dépend des activités psychiques humaines qui motivent l'émergence de ces formations. Toutefois, Reinach ne se contente apparemment pas de faire du Lipps. Son travail semble même passablement original comparé à l'œuvre de son directeur de thèse.

\section{Platonisme et antipsychologisme dans les Fondements a priori du droit civil}

Dans ses travaux ultérieurs, en particulier dans son texte sur le droit civil, Reinach retirera à la psychologie son monopole d'explication théorique du droit. Plus précisément, il distinguera le droit positif du droit a priori. La discipline qui s'occupe du droit a priori est la phénoménologie. Celle-ci a un sens bien précis pour Reinach: elle est une méthode invitant le philosophe à se tourner vers les "phénomènes ", c'est-à-dire les "choses mêmes ", ce par quoi Reinach entend les «essences". Plus précisément, ce que le philosophe doit saisir, ce sont des "connexions d'essences» (Wesenszusammenhänge) ${ }^{14}$. Ces connexions d'essences sont qualifiées d' "états de choses" (Sachverhalte), à ne pas confondre avec les «propositions» (Sätze). Comme le souligne Reinach:

Stumpf, Bergmann, Bolzano aussi - tous les autrichiens confondent constamment les propositions et les états de choses (Reinach I989 [I9I3/4], p. 526 n. I).

La «proposition» (Satz) est ce grâce à quoi le jugement "pose» (setzen) un état de choses. Faut-il comprendre que la proposition est le "contenu» du jugement, au sens où elle est ce qui donne au jugement sa direction intentionnelle? Reinach distingue explicitement proposition et contenu de jugement. Le contenu de jugement est alors peut-être le pendant psychologique de la proposition, celle-ci garantissant l'équivalence entre les contenus de deux jugements numériquement distincts. Quant à l'état de choses, il est le "corrélat intentionnel » du jugement. Selon Reinach, il peut subsister ou ne pas subsister, et il est ce à quoi reviennent les modalités, comme la possibilité ou la nécessité15. Or les connexions d'essences sont des «états de choses universels et nécessaires ", dits aussi «états de choses a

14. Voir notamment Reinach I989 [I9I4], 425.38-40, et I989 [I9I3b], 448.28-450.36.

15. Sur les états de choses, voir notamment Reinach I989 [I9II], I I 2.7-II7.22. Sur la distinction entre proposition et contenu de jugement, voir Reinach I989 [I9I3 b], 459.20-22. Pour une étude de la théorie reinachienne des états de choses, voir Salice 2009. 
priori». L'a priori, pour Reinach ne s'applique pas en premier lieu aux propositions, aux jugements ou à la connaissance, mais aux états de choses. Les propositions, jugements ou connaissances sont a priori dans la mesure où les états de choses vers lesquels ils pointent sont a priori. À noter que l'a priori des connexions d'essences n'est pas, pour Reinach, "analytique", mais "synthétique »: dans les propositions concernées, le prédicat n'est pas inclus dans le sujet, et le contraire de ces propositions, c'est-à-dire leur négation, n'implique pas de contradiction logique. D'après Reinach, Kant a limité l'a priori synthétique aux mathématiques et aux sciences de la nature, alors qu'il s'applique en fait à toutes les disciplines possibles, y compris au droit. Comme l'a remarqué Husserl, que Reinach suit, l'a priori n'est pas confiné à ce qui est formel, mais inclut aussi le matériel, jusqu'aux couleurs et aux sons, qui ont eux aussi leurs lois d'essence ${ }^{16}$. Toujours dans la continuité de Husserl, Reinach souligne à cet égard l'existence d'une psychologie a priori, qui n'est pas une science empirique de la psyché humaine, mais porte sur l'essence même des divers actes possibles et concerne donc tout être psychique possible (Reinach I989 [I9I4], 532.40-535.15) ${ }^{17}$.

Les états de choses universels et nécessaires, ou "connexions d'essences ", sont, comme leur nom l'indique, fondés dans des «essences ${ }^{18}$. Les essences elles-mêmes sont des objets idéaux, ou "atemporelles» (außerzeitlich); elles n'existent pas dans le temps, contrairement aux choses dites "réelles ». Il y a ainsi deux sortes d'existence, l'existence réelle et l'existence idéale $^{19}$. Reinach n'affirme apparemment pas explicitement que les états de choses sont de nature idéale ${ }^{20}$. Toutefois, il soutient que les connexions d'essence ont un «être éternel » (ewiges Sein) et dit par ailleurs qu'il y a des états de choses "atemporels» (außerzeitlich), ceux précisément fondés sur des idéalités (Reinach I989 [I9I3a], 205.I8-2I). Les états de choses atemporels semblent se distinguer de ceux qui sont "uniques» et "fortuits", ceux-ci étant constitués d'individus réels et étant donc temporels (zeitlich) ${ }^{21}$. Ainsi, on pourrait peut-être reconnaître une distinction en ce qui concerne la subsistance elle-même entre celle, temporelle, des états de choses constitués de réalités et celle, atemporelle, des états de choses fondés sur des

16. Cf. notamment Reinach I989 [I9I3a], I43.35-I46.7 et I48.3 I-I49.5, I989 [I9I4], 544.4I-545.3 et 546.28-547.I, ainsi que Kant I9II, 30.20-39. I9 et Husserl I984, 255.27258.24. Sur le fait que les connexions d'essence sont des états de choses, voir l'affirmation explicite dans Reinach I989 [I9II], 70.20-26.

17. Pour les développements husserliens les plus clairs d'une psychologie eidétique, voir Husserl I968.

18. Cf. à nouveau Reinach I989 [I9I3a], I43.35-I46.7.

19. Cf. notamment Reinach I989 [19I3 b], 420.40-42I.I3.

20. Baltzer-Jaray 2009 , qui critique Smith I987. Sur les «lois d'essence» dans la tradition phénoménologique de Munich et Göttingen plus généralement, voir Fréchette 2015.

21. À ce sujet, cf. Reinach I989 [I9IO], 35I.25-28 et I989 [I9I3a], I43.35-I44.30 et 205.I8-2I. 
idéalités. Ces états de choses peuvent donc, par extension, être qualifiés d'«idéaux ${ }^{22}$ ».

Pour Reinach, les états de choses a priori sont directement saisissables par ce qu'il qualifie de "vision des essences» ou Wesenschau:

[...] toutes les connaissances aprioriques sans exception sont susceptibles d'une évidence irréfutable, c'est-à-dire d'une intuition qui soit ultimement donatrice de leur teneur. Ce qui se fonde dans l'essence des objets peut, dans la vision d'essence, être élevé au statut de donnée ultime (Reinach I989 [I9I4], 546.6-Io; trad. Dewalque).

Or l'admission d'une sphère d'idéalités atemporelles et l'invitation à les saisir de manière intuitive se confondent, d'après Reinach, avec le projet philosophique de Platon. Comme le soutient Reinach, dans des passages où il décrit la méthode à suivre en philosophie:

Socrate a réalisé une analyse de signification lorsqu'il posait sa question dans les rues d'Athènes: tu parles de ceci ou de cela, mais qu'entends-tu par là? Il s'agit ici d'éclairer l'obscurité et les contradictions de ce qui est signifié — un procédé qui par ailleurs n'a strictement rien à voir avec la définition, ou même avec l'induction. Platon, en revanche, ne part pas du mot et de la signification; son but est la vision directe des Idées, la saisie non médiatisée des essentialités en tant que telles (Reinach I989 [I9I4], 542.3 I-37; trad. Dewalque)23.

Les visions d'essence doivent elles aussi être acquises laborieusement - et ce travail est placé sous l'image que Platon a dépeinte dans le Phèdre, l'image des âmes qui doivent gravir le ciel avec leurs attelages pour contempler les Idées (Reinach I989 [I9I4], 550.2I-24; trad. Dewalque).

Le texte de Reinach de I9I 3 sur les fondements a priori du droit civil a été publié dans le premier volume du Jahrbuch für Philosophie und phänomenologische Forschung, la revue de Husserl. Dans ce même numéro sont parues non seulement les Ideen I, mais aussi des essais d'autres phénoménologues de Munich et de Göttingen, à savoir Alexander Pfänder et Moritz Geiger. Or, lorsqu'il se félicite de la publication de ce volume, Reinach le fait en ces termes:

$<$ Que l'on pense aux> tentatives du nouveau «Jabrbuch» qui se dirigent vers $<$ la $>$ découverte des régularités légales a priori dans divers directions et domaines. $<$ Un $>$ mouvement en direction de la théorie des Idées de Platon. La

22. Comme le fait, outre Smith I987, Pradelle 2016.

23. Reinach fait sans doute référence ici aux «premiers dialogues» ou «dialogues socratiques ", dans lesquels Platon, plutôt que d'élaborer sa propre philosophie, relate surtout les propos de Socrate. Il s'agit des dialogues suivants: Charmide, Criton, Euthydème, Euthyphron, Gorgias, Hippias majeur, Hippias mineur, Ion, Lachès, Lysis et Protagoras. Sur ces dialogues, voir Kraut 2017. L'idée que Socrate ne procède ni à des définitions ni à des inductions s'oppose à l'interprétation d'Aristote, Mét. M, 4, I078b27-32; pour un renvoi à Aristote, voir Reinach I989 [I9I3b], 468.28-40. Je remercie Leone Gazziero pour la référence à Aristote. 
phénoménologie <comme> retour à Platon! (Reinach I989 [I9I3b], 44I.9-I2; ajouts des éditeurs) ${ }^{24}$.

Toutefois, Reinach ne prétend pas adopter l'entier de la doctrine platonicienne. Le principal reproche qu'il adresse à Platon est d'avoir assimilé la saisie des Idées à une perception sensible. Par contraste, Reinach, s'inspirant de Husserl, se dit doté d'un type particulier de faculté perceptive, non pas sensible, mais "catégoriale» et permettant de saisir les idées "in via» ${ }^{25}$. Ainsi, pour Reinach:

Platon a découvert les Idées et leurs connexions. Mais il n'est pas parvenu à rendre compte de la manière dont ces Idées et leurs connexions sont saisies $<$ et $>$ connues. <D'où son> hypothèse <d'une $>$ expérience durant l'existence pré-mondaine. Mais tout cela est en fait $<$ un $>$ retour en arrière vers $<$ l'>empirisme. Pour nous, les Idées et les relations <sont> toujours données dans une perception catégoriale. Mais la position de Platon consistait à soutenir que tout devait par principe être donné dans la perception sensible, <et> puisque cela ne l'était pas dans cette vie, <alors> nécessairement dans <une> vie extramondaine, donc ici dans une <perception> pré-mondaine. $<\mathrm{Un}>$ bien étrange empirisme idéaliste! Il ne reconnaissait pas d'actes de perception non sensibles dans cette vie. Ainsi, <un> domaine empirique au-delà de ce monde est admis - un tournant métaphysique vers <un> autre monde de l'expérience (Reinach I989 [I9I3b], 429.3 I-42) ${ }^{26}$.

On trouve dans le cours de Reinach de i9 so sur Platon une idée semblable: la théorie de l'anamnèse, qui présuppose une acquisition cognitive des Idées avant l'incarnation, brouille la différence entre connaissance a priori et empirique (Reinach 2000).

Je ne discuterai pas ici la justesse de l'interprétation de Platon par Reinach. Je tiens seulement à souligner que c'est Reinach lui-même qui base sa théorie de l'a priori et des idéalités sur Platon, tout en rejetant le «tournant métaphysique» platonicien consistant à poser un monde des Idées compris comme lieu empirique, c'est-à-dire d'expérience.

Reinach, ayant ancré sa théorie dans les thèses de Platon, peut légitimement se revendiquer de Husserl, du moins prima facie. Comme indiqué dans l'introduction, Husserl, dans les Recherches logiques, admet des entités idéales, auxquelles il attribue une certaine existence ${ }^{27}$. Il parle même, en ce qui les concerne, d'un "règne intemporel des Idées » (Husserl I975, I 36.6-9, cité dans Dubois I995, p. I03). Dans un projet de préface à la nouvelle

24. La référence à Platon est Phèdre 247b.

25. Sur l'inadéquation de cet élargissement du sens de l'expression «intuition catégoriale ", réservée, chez Husserl, à la saisie des essences formelles, cf. Pradelle 20I 5, p. 55, citant Husserl I984, 7II.I 5-7I4.5.

26. Reinach pense probablement ici à Ménon $8 \mathrm{Ic}-\mathrm{d}$, au Banquet 2I $2 \mathrm{a}$ ou encore à Phèdre 249b-c.

27. Voir notamment Husserl I984, I30.9-10. 
édition de son texte, projet daté de I9I3, Husserl affirme explicitement avoir endossé une variante du platonisme ${ }^{28}$. Or, lorsque Husserl défend, dans les Recherches logiques, l'existence d'idéalités, il met immédiatement son lecteur en garde contre toute "hypostase métaphysique " qui placerait

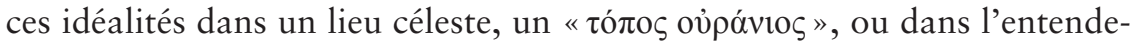
ment divin (Husserl I984, I06.I 5-I9). Le refus de Husserl de localiser ces entités évoque les remarques de Reinach sur le dédoublement du monde empirique chez Platon. Au vu des textes cités, il semble donc plausible que Reinach, pour ce qui est de son platonisme "non métaphysique ", s'inspire de Husserl. En somme, pour Reinach, la phénoménologie peut, à la suite de Husserl, être vue comme un retour à Platon, à condition d'éviter tout «empirisme».

Reste dès lors à se demander comment ce "platonisme» s'applique au droit. Reinach considère que les "formations juridiques» ont elles aussi leurs essences: il y a des essences de la prétention, de l'obligation ou de la promesse. Cela vaut non seulement pour le droit civil, auquel Reinach s'intéresse en particulier, mais pour tous les domaines du droit. Par contre, les essences en question sont totalement indépendantes des divers droits positifs, qui ne les créent en aucun cas (Reinach I989 [I9I3a], I43.35-I46.7). Quand Reinach en vient à étudier la nature des diverses formations de droit civil, notamment celles de la prétention et de l'obligation, ainsi que celle de la promesse, il procède à des analyses particulièrement originales. Tout d'abord, les formations juridiques que sont la prétention et l'obligation ont un statut ontologique particulier: il ne s'agit de choses ni physiques ni psychiques. La prétention et l'obligation ne sont néanmoins pas des entités idéales. Elles naissent en effet dans le temps et ont une certaine durée. À noter qu'il en va ainsi pour les prétentions et obligations individuelles, qu'il convient de distinguer de leurs essences; celles-ci, comme toutes les essences, sont atemporelles et donc idéales. En guise d'exemple d'état de choses a priori juridique, Reinach donne celui-ci: «La prétention à une prestation déterminée cesse dans l'instant même où cette prestation est accomplie." Cette proposition n'est pas analytique, puisque «dans le concept de prétention n'est contenu en aucun sens possible le fait qu'elle puisse prendre fin en certaines circonstances ». S’il est plutôt évident que les prétentions et obligations ne sont pas des entités physiques, Reinach prend soin de les distinguer des «vécus» (Erlebnisse) dont elles pourraient être éventuellement accompagnées. Ces formations juridiques ne sont en rien psychiques: les vécus semblent se modifier avec le temps, tandis que les prétentions et obligations peuvent demeurer identiques durant des années, de même qu'un sujet «dans un état de profonde inconscience» reste porteur de ses prétentions et obliga-

28. Husserl I939, p. I28-I30, et 2002, 297.20-299.4, où il est fait référence à l'interprétation de Platon par Lotze (I9I2). Contre l'interprétation platonisante de Husserl, voir notamment Seron 20I2, mentionné dans l'introduction. 
tions (Reinach I989 [I9I3a], I45.25-I46.7 et I48.I2-30; trad. de Calan). De même, non seulement les prétentions et obligations, chez Reinach, ne sont pas psychiques, mais certains droits se fondent sur des entités non psychiques. Il en va ainsi des droits de propriété, par exemple le droit de disposer de la chose, qui découlent directement de la relation de propriété entre le propriétaire et la chose, relation qui est elle-même naturelle, indépendante du droit positif et primitive (Reinach I989 [I9I3a], I89.25-204.9) ${ }^{29}$.

Quant à la promesse, Reinach la qualifie d' "acte social». Cette dernière notion a souvent été vue comme une anticipation de la théorie dite des "actes de langage " (speech acts), c'est-à-dire des "actes qui peuvent (bien qu'ils ne doivent pas) être accomplis en disant qu'on le fait ", comme c'est le cas des promesses, mais aussi des avertissements ou des excuses ${ }^{30}$. Un acte social, pour Reinach, est une activité psychique spontanée, pourvue d'un contenu intentionnel, s'adressant à autrui et requérant d'être perçue pour être accomplie. Ainsi, un ordre est spontané (on ne le subit pas, mais l'initie), il a un contenu intentionnel ( «que tu adoptes tel comportement»), il s'adresse à autrui (on ne se donne pas d'ordre à soi-même, sauf à se dédoubler) et doit être perçu pour être efficace (un ordre qui résonne dans le vide n'a pas été donné). Du fait de la nécessité d'être perçus, les actes sociaux requièrent un revêtement physique, le plus souvent langagier. Ces actes ont donc, comme le dit Reinach, une âme et un corps. Toutefois, il est possible d'imaginer des cas où ils seraient dépourvus de revêtement corporel, par exemple entre êtres capables de mutuellement percevoir leurs pensées par la pensée. Un autre exemple donné par Reinach, moins angélologique, mais tout aussi religieux, est celui de la prière silencieuse, Dieu ayant le pouvoir de lire dans les pensées (Reinach I989 [I9I3a], I 58.6I6I.I3). Tandis que Reinach s'évertue à distinguer, d'une part, prétentions et obligations, de l'autre, vécus, il affirme par contre que les actes sociaux, eux, sont "psychiques" (Reinach I989 [I9I3a], 276.35-36). Est-ce là une concession au psychologisme? Nous allons y revenir dans les paragraphes qui suivent. En tous les cas, le fait que les actes sociaux soient psychiques ne fait pas de leur résultat, à savoir les prétentions et obligations, quelque chose de psychique. Prétentions et obligations, bien que découlant ici d'entités psychiques, contrairement à ce qui est le cas des droits de propriété, restent néanmoins elles-mêmes non psychiques. À noter que, pour Reinach, le droit positif est également un acte social, plus précisément un ensemble de «dispositions» (Bestimmungen), c'est-à-dire d'actes sociaux

29. Sur cette question, voir Massin 2017.

30. Sur les actes de langage chez Reinach, voir Mulligan 1987, Ambroise 2005 et Picavet 2008. Pour la définition des actes de langage, ainsi que pour les exemples, voir Green 20 I4. Pour une confrontation critique avec Austin, voir Benoist 2008, qui reproche notamment à Reinach sa tendance proprement "philosophante " à ontologiser les prétentions et obligations qui découlent des actes sociaux, contrairement à Austin. 
qui «posent que quelque chose doit être» (Reinach I989 [1913a], 238.2625 I.37; trad. de Calan) ${ }^{31}$.

Qu'en est-il dès lors du psychologisme dans le texte de I9I3, de la critique de Lipps et des éventuels reniements par Reinach de ses écrits de jeunesse? Reinach reproche effectivement à Lipps d'être psychologiste juridique (Reinach I989 [I9I3a], I79.I9-I83.23). Tout d'abord, il rejette la thèse de Lipps voulant que la promesse soit une déclaration de volonté: déclarer sa volonté de faire quelque chose et s'engager à le faire, ce sont deux choses différentes, la seconde étant un acte social particulier. Cette critique n'est néanmoins pas motivée par le fait que la promesse est analysée au moyen d'une catégorie psychologique puisque, pour Reinach luimême, comme indiqué ci-dessus, la promesse est un acte psychique. Par contre, selon Reinach, la prétention et l'obligation ne sont pas des actes psychiques et n'en sont pas même des signes. Analyser le contenu de la notion d'obligation par la notion d'acte psychique, comme le fait Lipps, c'est être psychologiste:

Maintenons-le fermement: se fondent en [l'acte de déclaration] prétention et obligation et non un vécu ou un sentiment d'être autorisé à. Naturellement, ceux-ci peuvent apparaître dans de nombreux cas, mais c'est tout à fait inessentiel pour la question qui nous occupe. Et ici nous en arrivons au point où les doutes suscités par la conception de Lipps sont les plus importants. Si nous [...] admettons que par la déclaration de volonté naît du côté du locuteur un se sentir obligé et du côté du destinataire un se sentir autorisé - qu'est-ce qu'on a gagné par là ? Si la prétention et l'obligation apparaissent, voilà ce que nous voulons savoir. Ces vécus ne peuvent nous offrir pour cela aucun remplacement. Ou peut-être doivent-ils être le signe du fait que les prétentions et obligations objectives existent réellement? Ceci constituerait un signe bien peu fiable. Nous voyons bien que des prétentions et des obligations existent souvent sans être relevées dans des vécus correspondants; et qu'il y a d'un autre côté des illusions où nous nous sentons obligés ou autorisés sans l'être effectivement. Pour les rapports d'essence objectifs qui sont établis entre les promesses d'un côté et les prétentions et obligations de l'autre, ces vécus peuvent nous offrir aussi peu de substitut ou de garantie que par exemple un vécu de «nécessité de pensée» peut nous offrir une garantie de la validité des lois objectives de la logique. On caractérise l'effort consistant à expliquer les règles de la logique par retour sur des vécus plutôt que de les élucider par leur propre analyse et de les rendre évidents, comme étant de nature psychologiste. On devra donc parler ici également de psychologisme quand on entreprendra d'expliquer l'objectivité des rapports sociaux dotés d'une légalité essentielle par un recours aux vécus qui sont absolument sans intérêt pour l'existence de ces rapports (Reinach I989 [I9I3a], I82.40-I83.23; trad. de Calan, légèrement modifiée, mon ajout).

31. Pour une discussion critique de la théorie a priori du droit chez Reinach, en faveur d'un primat du droit positif, ou des "dispositifs normatifs informels ", et donc d'une certaine forme de conventionnalisme, voir à nouveau Benoist 2008. 
Ainsi, Lipps est psychologiste parce qu'il analyse le contenu de notions non psychiques en termes d'actes psychiques et assimile ainsi les essences non psychiques à des essences psychiques. Il s'agit là d'un psychologisme fondé sur la psychologie descriptive. Toutefois, il convient de rappeler que Reinach ne rejette pas toute analyse du contenu de notions juridiques en termes d'actes psychiques, puisqu'il comprend les actes sociaux, y compris la promesse, comme étant de nature psychique ${ }^{32}$. Donc, le psychologisme juridique critiqué ici par Reinach n'est pas la théorie assimilant n'importe quelle formation juridique à une entité psychique, mais seulement celles qui... ne sont pas psychiques. Un point tout à fait capital, néanmoins, est l'insistance de Reinach sur le fait que l'analyse psychologique des notions juridiques n'implique pas leur limitation à la psychologie humaine. En effet, comme il le souligne:

Quel que soit le sujet dans lequel une promesse se réalise, que ce soit un ange, un diable ou Dieu lui-même qui se fassent des promesses, prétentions et obligations naissent également chez les anges, les diables ou chez Dieu - pour autant qu'ils puissent réellement promettre et entendre les promesses. Car notre rapport ne se fonde que sur les promesses en tant que promesses; non sur le fait qu'elles sont réalisées par des individus qui se tiennent sur deux jambes et sont appelés humains (Reinach I989 [19I3a], I88.33-39; trad. de Calan $)^{33}$.

De toutes les manières, il y a, pour Reinach, une psychologie a priori, qui n'est dès lors pas une science empirique de la psyché humaine, mais vaut pour tout être psychique possible (Reinach I989 [I9I4], 532.4O-535.I5). C'est vers elle et son domaine d'essences qu'il convient de se tourner lorsque l'on s'interroge sur la nature des actes psychiques requis dans l'explication du droit. En somme, si Reinach reproche à Lipps d'analyser des formations juridiques non psychiques à l'aide de notions de psychologie descriptive, et donc en ce sens d'être psychologiste, il admet lui-même que certaines entités juridiques doivent être analysées de la sorte; toutefois, ce n'est jamais la psychologie descriptive humaine qui est à mobiliser, mais la phénoménologie des essences psychiques et de leurs relations. En d'autres termes, Reinach rejette de manière explicite tout psychologisme juridique anthropologique.

32. On pourrait du reste s'étonner que Reinach hésite à faire découler prétentions et obligations de la promesse insincère (Reinach I989 [I9I3a], I68.25-I69.3). Usuellement, une promesse est basée sur la volonté, chez celui qui promet, d'effectuer telle ou telle action, sans que la promesse, comme acte social, se confonde avec cette volonté. Or, dans la promesse insincère, celui qui promet n'a pas la volonté sous-jacente d'effectuer l'action en question. Mais n'est-ce pas une sorte de résidu de psychologisme (génétique) qui mène Reinach à se demander si des prétentions et obligations naissent d'une promesse insincère? L'efficacité de l'acte social ne devrait en rien dépendre de ce qui se passe «dans la tête» de celui qui promet.

33. Voir également Reinach I989 [I9I3a], 275.33-278.4, pour des considérations similaires. 
Si l'on compare le texte de I9I3 à celui de 1905 , les rapports entre droit et psychologie subissent des changements importants. Tout d'abord, la psychologie perd son monopole explicatif théorique sur le droit. Ce dernier a sa portion propre de monde des Idées, perçue "catégorialement» par le philosophe, plus précisément par le phénoménologue. En outre, les essences juridiques ne sont en rien dépendantes, pour leur émergence ou leur teneur, de l'existence d'êtres humains. Il convient de distinguer, d'une part, la genèse du droit positif ou son existence temporelle sous forme de "dispositions" (Bestimmungen) et, d'autre part, l'existence idéale des essences juridiques. De même, faudrait-il ajouter, l'interprétation et l'application du droit, si elles peuvent requérir des connaissances en psychologie, éventuellement en psychologie humaine, se distinguent totalement de la vision des légalités juridiques a priori. Par ailleurs, même dans les cas où le contenu des notions juridiques est analysé en termes psychiques, il ne saurait être question de psychologie humaine: c'est la psychologie a priori qui est à mobiliser, laquelle possède son propre domaine d'essences. Ainsi, le psychologisme anthropologique est intégralement exclu du traité sur le droit civil.

\section{Conclusion}

Le jeune Reinach, dans sa thèse sur la causalité en droit pénal, accorde une place importante à la psychologie: les origines et, surtout, la teneur des systèmes juridiques dépendent de la psyché humaine, de ses croyances, intuitions morales, etc., ce qui consacre dès lors un psychologisme juridique anthropologique basé sur des considérations de psychologie génétique; en outre, l'interprétation et l'application du droit sont impossibles sans connaissances psychologiques; quant à la "philosophie du droit ", elle se limite à des discussions éthiques sur le droit juste. Quelques années plus tard, les choses changent radicalement: une portion du monde des Idées est réservée aux essences du droit et à leurs connexions, accessibles au philosophe par Wesenschau. Parallèlement au psychologisme en logique, qui consiste à faire dépendre les règles de la logique de celles de la pensée, qu'elle soit humaine ou non, il y a, pour le Reinach de la maturité, un psychologisme juridique, qui consiste à analyser indument en termes d'actes psychiques le contenu de notions juridiques non psychiques et donc à assimiler des essences psychiques et non psychiques; il s'agit là d'un psychologisme non anthropologique fondé sur la psychologie descriptive. Cela n'empêche pas certaines entités juridiques d'être psychiques. Toutefois, lorsqu'elles le sont, il n'est pas question de mobiliser la psychologie descriptive humaine afin de les expliquer, puisque les lois psychiques concernées sont là aussi des lois d'essence, qui valent pour toute psyché possible. En somme, le psychologisme juridique anthropologique doit être intégralement banni.

Si les thèses du jeune Reinach semblent subir l'influence de Lipps, qui fait jouer un rôle explicatif important à la psychologie, non seulement en logique, mais aussi en philosophie du droit, les travaux reinachiens plus 
tardifs s'inspirent de Husserl: ce dernier oppose au psychologisme, c'est-àdire à l'explication des lois de la logique par les lois de la pensée humaine, une théorie platonicienne, suivant laquelle la logique est faite de lois idéales, ancrées dans un monde des Idées. Reinach, glissant de Lipps à Husserl, passe du psychologisme de l'un au platonisme de l'autre. La grande différence avec la critique husserlienne du psychologisme logique concerne la dimension relativiste: pour Reinach, "psychologisme » n'équivaut pas nécessairement à "anthropologisme"; toutefois, même en l'absence d'implications relativistes, le psychologisme peut poser un problème, non seulement en logique, mais aussi en droit, car il tend à analyser des catégories non psychiques en termes psychiques. C'est là sans doute une variante originale de la critique du "psychologisme ", qui n'a pas attiré l'attention des commentateurs. Or elle n'est pas dénuée d'intérêt. Elle semble en effet s'opposer par avance à toute tentative de "logique transcendantale", telle que Husserl l'entreprendra plus tard (Husserl I974), Reinach insistant sur l'indépendance des lois logiques à l'égard de toute activité psychique, que ce soit celle des humains ou d'autres sujets de pensée. La critique reinachienne peut surtout venir appuyer les théories objectivistes des entités sociales, par exemple les théories objectivistes de l'État, en insistant sur l'absence de marques psychiques dans les définitions de ces phénomènes ${ }^{34}$. Reinach semble notamment nous inviter à distinguer la genèse d'un phénomène social de sa définition, qui peut dépendre, quant à sa teneur, des entités psychiques dont elle émerge, mais sans que celles-ci n'intègrent la définition. Il y a sans doute là de quoi clarifier certaines discussions ${ }^{35}$.

\section{Bibliographie}

\section{Sources}

Aristote. Opera, E. Bekker (ed.), 2 vols., Berlin, Reimer, I83 I.

Bourdieu, Pierre. Choses dites, Paris, Les Éditions de Minuit, 1987.

Brentano, Franz. Deskriptive Psychologie, R. M. Chisholm, W. Baumgartner (eds.), Hamburg, Felix Meiner, 1982.

Husserl, Edmund. "Entwurf einer "Vorrede" zu den "Logischen Untersuchungen" (1913)", E. Fink (ed.), Tijdschrift voor Philosophie I, I939, p. I06-I33 et p. 3 I9-339.

—. Phänomenologische Psychologie. Vorlesungen Sommersemester 1925, Husserliana IX, W. Biemel (ed.), The Hague, Martinus Nijhoff, 1968.

34. Sur l'opposition entre objectivisme et subjectivisme dans les sciences sociales, voir notamment Bourdieu 1987.

35. Une première version de cet article a été présentée en 2017 dans le cadre du séminaire «Des platonismes en phénoménologie» dirigé par Alain Lernould, Claudio Majolino et Édouard Mehl à l'Université de Lille 3. Je remercie les participants pour leurs remarques, en particulier Leone Gazziero, Carla Di Martino et Claudio Majolino. Ma reconnaissance va également aux trois évaluateurs de cette revue pour leurs précieux commentaires. 
- Formale and transzendentale Logik. Versuch einer Kritik der logischen Vernunft, Husserliana XVII, P. Janssen (ed.), The Hague, Martinus Nijhoff, I974.

—. Logische Untersuchungen, Husserliana XVIII et XIX/I-2, U. Panzer (ed.), The Hague, Martinus Nijhoff, I975 et 1984 (traduction française: Recherches logiques, trad. H. Elie, A. L. Kelkel, R. Scherer, Paris, P.U.F, I959-I963).

- Logische Untersuchungen. Ergänzungsband. Erster Teil. Entwürfe zur Umarbeitung der VI. Untersuchung und zur Vorrede für die Nenauflage der Logischen Untersuchungen (Sommer 1913), Husserliana XX/I, U. Melle (ed.), The Hague, Kluwer, 2002.

Kant, Emmanuel. I787. Kritik der reinen Vernunft, $2^{\mathrm{e}}$ éd., in E. Kant, Gesammelte Schriften, vol. 3, Berlin, Reimer, I9I I.

Lipps, Theodor. "Die Aufgabe der Erkenntnistheorie und die Wundt'sche Logik", Philosophische Monatshefte I6, I880, p. 529-539.

—. Die ethischen Grundfragen, Hamburg-Leipzig, Leopold Voss, I 899.

—. Leitfaden der Psychologie, $2^{\mathrm{e}}$ éd., Leipzig, Wilhelm Engelmann, I906a.

—. "Der Begriff der Strafe", Monatsschrift für Kriminalpsychologie und Strafrechtsreform 3, I906b, p. 279-309.

Lotze, Hermann. Logik (System der Philosophie I). Drei Bücher vom Denken, vom Untersuchen und vom Erkennen, G. Misch (ed.), Leipzig, Felix Meiner, I9I 2.

Pfänder Alexander. Einführung in die Psychologie, Leipzig, J. A. Barth, I904.

Reinach, Adolf. I905. Über den Ursachenbegriff im geltenden Strafrecht, in A. Reinach, Sämtliche Werke, K. Schuhmann, B. Smith (eds.), vol. I, Munich, Philosophia, I989, p. I-43.

—. I910. "Notwendigkeit und Allgemeinheit im Sachverhalt», in A. Reinach, Sämtliche Werke, K. Schuhmann, B. Smith (eds.), vol. I, Munich, Philosophia, I989, p. $35 \mathrm{I}-354$.

—. I9I I. "Zur Theorie des negativen Urteils", in A. Reinach, Sämtliche Werke, K. Schuhmann, B. Smith (eds.), vol. I, Munich, Philosophia, I989, p. 95-I 40.

- I9 з 3 a. Die apriorischen Grundlagen des bürgerlichen Rechtes, in A. Reinach, Sämtliche Werke, K. Schuhmann, B. Smith (eds.), vol. I, Munich, Philosophia, I989, p. I4I-278 (traduction française: Les fondements a priori $d u d r o i t$ civil, trad. R. de Calan, Paris, Vrin, 2004).

—. I9г3b. "Einleitung in die Philosophie», in A. Reinach, Sämtliche Werke, K. Schuhmann, B. Smith (eds.), vol. I, Munich, Philosophia, I989, p. 369$5 \mathrm{I} 3$.

—. I9I3/4. "Zum Begriff der Zahl», in A. Reinach, Sämtliche Werke, K. Schuhmann, B. Smith (eds.), vol. I, Munich, Philosophia, I989, p. 5I 5-529.

—. I9I4. "Über Phänomenologie", in A. Reinach, Sämtliche Werke, K. Schuhmann, B. Smith (eds.), vol. I, Munich, Philosophia, I989, p. 53 I-550 (traduction française: "Sur la phénoménologie», trad. A. Dewalque, in A. Reinach, Phénoménologie réaliste, Paris, Vrin, 2012, p. 33-62).

-. Platons Philosophie. Vorlesung SS 1910, in J. Seifert, Ritornare a Platone, Milan, Vita e Pensiero, 2000, p. I8I-24I.

\section{Autres références}

Ambroise, Bruno. «Le problème de l'ontologie des actes sociaux: Searle héritier de Reinach?", Les études philosophiques 72, 2005, p. 55-71. 
Baltzer-Jaray, Kimberly. "Adolf Reinach is not a Platonist », Symposium: Canadian Journal of Continental Philosophy I3, 2009, p. I00-I I 2.

Benoist, Jocelyn. "Synthétique a priori et normativité", in J. Benoist et J.-F. Kervégan (dir.), Adolf Reinach. Entre droit et phénoménologie, Paris, CNRS Éditions, 2008, p. 3I-49.

Cantegreil, Julien. «D’une voie phénoménologie en théorie du droit. Remarques sur le réalisme d'Adolf Reinach ", Les études philosophiques 72, 2005, p. 99-I I 2.

Chisholm, Roderick M. "Editor's Introduction", in R. M. Chisholm (ed.), Realism and the Background of Phenomenology, Atascadero (CA), Ridgeview, I960, p. 3-36.

Depraz, Nathalie, Michel Espagne et Mildred Galland-Szymkowiak (dir.). Théodor Lipps 1, Revue de métaphysique et de morale, 2017.

- (dir.), Théodor Lipps 2, Revue de métaphysique et de morale, 20 I 8.

Dewalque, Arnaud, et Maria Gyemant. "Introduction. Le tournant psychologique ", in M. Gyemant (dir.), Psychologie et psychologisme, Paris, Vrin, 201 5, p. 7-39.

Dubois, James M. Judgment and Sachverhalt. An Introduction to Adolf Reinach's Realism, Dordrecht, Kluwer, I995.

Fréchette, Guillaume. «Essential Laws ", in B. Leclercq, S. Richard et D. Seron (dir.), Objects and Pseudo-Objects. Ontological Deserts and Jungles from Brentano to Carnap, Berlin, De Gruyter, 2015, p. I43-I 66.

Green, Mitchell. "Speech Acts", in E. N. Zalta (ed.), Stanford Encyclopedia of Philosophy, Stanford, 20I4 [plato.stanford.edu/entries/speech-acts].

Halpérin, Jean-Louis. "Les orientations de la pensée juridique de langue allemande à l'époque des Fondements a priori du droit civil », in J. Benoist et J.-F. Kervégan (dir.), Adolf Reinach. Entre droit et phénoménologie, Paris, CNRS Éditions, 2008, p. 99-II2.

Kraut, Richard. "Plato", in E. N. Zalta (ed.), Stanford Encyclopedia of Philosophy, Stanford, 20I7 [plato.stanford.edu/entries/plato].

Kusch, Martin. Psychologism. A Case Study in the Sociology of Philosophical Knowledge, London, Routledge, I995.

Lavigne, Jean-François. "Psychologie et critique du psychologisme: à l'origine de la phénoménologie, la controverse Lipps-Husserl ", Revue de métaphysique et de morale, 201 8, p. 49-7I.

Loidolt, Sophie. Einführung in die Rechtsphänomenologie. Eine historisch-systematische Darstellung, Tübingen, Mohr Siebeck, 2010.

Massin, Olivier. "The Metaphysics of Ownership: A Reinachian Account ", Axiomathes 27, 2017, p. 577-600.

Mulligan, Kevin. «Promisings and Other Social Acts: Their Constituents and Structure", in K. Mulligan (ed.), Speech Act and Sachverhalt. Reinach and the Foundations of Realist Phenomenology, Dordrecht, Martinus Nijhoff, I987, p. 29-90.

Picavet, Emmanuel. "Formes sociales et actes sociaux. L'originalité du point de vue de Reinach ", in J. Benoist et J.-F. Kervégan (dir.), Adolf Reinach. Entre droit et phénoménologie, Paris, CNRS Éditions, 2008, p. I79-I 97.

Pradelle, Dominique. "De Husserl à Heidegger: intentionnalité, monde et sens ", Discipline Filosofiche XXV, 2, 20I 5, p. 36-68.

—. "La critique de la subjectivation de l'a priori chez Reinach ", Philosophiques I 28, 20I6, p. 3-I9. 
Salice, Alessandro. Urteile und Sachverhalte. Ein Vergleich zwischen Alexius Meinong und Adolf Reinach, Munich, Philosophia Verlag, 2009.

—. "The Phenomenology of the Munich and Göttingen Circles », in E. N. Zalta (ed.), Stanford Encyclopedia of Philosophy, Stanford, 201 5, [plato.stanford. edu/entries/ phenomenology-mg].

Seron, Denis. "Phénoménologie et objectivisme sémantique dans les Recherches logiques de Husserl ", in B. Collette-Ducic et B. Leclercq (dir.), L'Idée de l'Idée. Éléments de l'histoire d'un concept, Louvain, Peeters, 20I 2, p. 2I 5-238.

Smith, Barry. "On the Cognition of States of Affairs", in K. Mulligan, Speech Act and Sachverhalt: Reinach and The Foundations of Realist Phenomenology, Dordrecht, Martinus Nijhoff, I987, p. I89-225. 